\title{
INFLUENCIA DEL USO DE MALLA PERLA Y ROJA EN LAS CONDICIONES MICRO-CLIMÁTICAS, DAÑO POR SOL Y DESARROLLO DE COLOR DE FRUTOS EN HUERTOS DE MANZANOS CVS. GALA Y FUJI ${ }^{1}$
}

\author{
CATALINA UMANZOR ${ }^{2}$, RICHARD MAURICIO BASTIAS IBARRA ${ }^{3}$, \\ ROSEMARIE WILCKENS ${ }^{4}$, CELERINO QUEZADA ${ }^{5}$
}

RESUMEN- El daño por sol y la falta de color de frutos producida por el exceso de radiación solar y altas temperaturas es el mayor problema productivo en los huertos de manzano en Chile. El objetivo de esta investigación fue evaluar el uso de mallas de colores como herramienta para el control de daño por sol y mejorar la coloración en manzanas. Durante la temporada 2012-13 y 2013-14 un huerto de manzanos 'Fuji' y 'Gala' fue cubierto con mallas de color Roja y Perla al 20\% de sombreado, dejando árboles sin cubrir como control. Se realizó un registro de la temperatura del aire y fruto $\left({ }^{\circ} \mathrm{C}\right)$, humedad relativa $(\%)$ y radiación fotosintéticamente activa total $\left(\mathrm{PAR}_{\text {total }}\right)$ y difusa $\left(\mathrm{PAR}_{\text {difusa }}\right)\left(\mu \mathrm{mol} \mathrm{m}^{-2} \mathrm{~s}^{-1}\right)$. Al momento de cosecha se cuantificó la incidencia de daño por sol con escala visual (\%) y el color de frutos a través de un equipo Color Hunter. En promedio, para ambas temporadas, la malla Roja redujo la transmisión de $\mathrm{PAR}_{\text {total }}$ en un $21 \%$, mientras que la malla Perla en un $17 \%$. La cantidad PAR difusa fue en promedio un $15 \%$ superior bajo la malla Perla que la malla Roja, incrementando con ello el desarrollo de color rojo de frutos en el cultivar Fuji. Durante la temporada 2012/13 ambas mallas redujeron en un $26 \%$ y $44 \%$ el daño por sol en frutos para los cultivares Gala y Fuji, respectivamente. Durante la temporada 2013/14 las mallas no redujeron significativamente el daño por sol en los frutos. Los resultados de este estudio sugieren que la malla Perla provee de mejores condiciones de luz para el desarrollo de color rojo de frutos en huertos de manzanos. No obstante, bajo las condiciones climáticas de Chile, ambas mallas (Roja y Perla) presentan una efectividad solo relativa para el control de golpe de sol en manzanas.

Términos para indexar: Mallas de color, condiciones de luz, color de frutos, golpe de sol, Malus domestica Borkh.

\section{INFLUENCE OF USING OF PEARL AND RED NETS ON MICRO-CLIMATE CONDITIONS, FRUIT SUN DAMAGE AND FRUIT COLOR DEVELOPMENT IN 'GALA' AND 'FUJI' APPLE ORCHARDS}

\begin{abstract}
Sun damage and the lack of color of fruits produced by excessive solar radiation and high temperatures is the biggest production problem in apple orchards in Chile. The objective of this research was to evaluate the use of colored nets as a tool for control of sun damage and improve color on apples. During the 2012-13 and 2013-14 season 'Fuji' and 'Gala' apple orchard was covered with Red and Pearl nets at 20\% of shading, leaving trees without covering as control. The air and fruit temperature $\left({ }^{\circ} \mathrm{C}\right)$, relative humidity $(\%)$, total $\left(\mathrm{PAR}_{\text {total }}\right)$ and diffuse $\left(\mathrm{PAR}_{\text {difusse }}\right)$ photosynthetically active radiation $\left(\mu \mathrm{mol} \mathrm{m} \mathrm{m}^{-2} \mathrm{~s}^{-1}\right)$ were recorded. At the harvest moment, sun damage by visual scale (\%) and color by a Color Hunter equipment were measured in fruits. On average, for both seasons, the Red net reduced the PAR total transmission by $21 \%$, while the Pearl net was up to $17 \%$. The amount of PAR difuse was on average $15 \%$ higher under Pearl than Red net, thereby increasing the development of red color in fruits at Fuji cultivar. During the 2012/13 season both net reduced by $26 \%$ and $44 \%$ the sun damage in fruit for the Gala and Fuji cultivars, respectively. During the 2013/14 season the nets did not significantly reduce sun damage in the fruits. The results of this study suggest that the Pearl net provides better light conditions for the development of red color of fruits in apple orchards. However, under the climatic conditions of Chile, both nets (Red and Pearl) have only relative effectiveness for controlling sun damage on apples.
\end{abstract}

Index terms: Colored nets, light conditions, fruit color, sunburn, Malus domestica Borkh.

\footnotetext{
(Trabajo 183-15). Recibida el 20 de julio el año 2015. Aceptado el 2 de de mayo de 2016.

${ }^{2}$ Ingeniero Agrónomo, Candidato a Magíster en Cs. Departamento de Producción Vegetal, Universidad de Concepción. E-mail: mumanzor@udec.cl

${ }^{3}$ Ingeniero Agrónomo, Dr. Profesor Asociado. Departamento de Producción Vegetal, Universidad de Concepción. E-mail: ribastias@ udec.cl

${ }^{4}$ Biólogo, Dr. Profesor Asociado. Departamento de Producción Vegetal, Universidad de Concepción. E-mail: rwilcken@udec.cl ${ }^{5}$ Ingeniero Agrónomo, Mg. Cs. Profesor Asociado. Departamento de Suelos y Recursos Naturales, Universidad de Concepción. E-mail: cequezad@udec.cl
} 


\section{INTRODUCCION}

Chile es actualmente uno de los más eficientes productores del mundo en el mercado de las manzanas, (ocupando el séptimo lugar del mercado), y con una productividad de $25,7 \mathrm{t} \mathrm{ha}^{-1}$, por lo que es considerado líder mundial en competitividad en la industria. La producción de manzanas en Chile se concentra entre las regiones de O'Higgins y Maule que representan el $80 \%$ de la superficie nacional, existiendo además un incremento en las plantaciones en la zona centro-sur (Regiones del Bío Bío y La Araucanía) del orden de $38,6 \%$ en seis años (BAEZA y NAVARRO, 2008). Bajo estas condiciones climáticas, los productores enfrentan recurrentes pérdidas económicas por quemadura de sol en la fruta que en variedades susceptibles representa un $40 \%$ de descarte de fruta en los huertos de Chile (VALENZUELA y MUÑOZ, 2011). Para mitigar los efectos ocasionados por el sol se ha buscado solucionar el problema mediante la aspersión de agua de riego sobre los árboles y fruta y formulaciones en base a caolinas y films translúcidos para reducir la absorción de radiación en frutos.

El uso de mallas es una técnica que se está masificando entre los productores de Chile como estrategia para mitigar las pérdidas de golpe de sol. Los antecedentes existentes a la fecha indican que con el uso de esta tecnología es posible disminuir el daño por sol entre un $10-20 \%$. Sin embargo, dependiendo del cultivar y condición climática, la efectividad de estas mallas es solo relativa; existen años en que prácticamente no son capaces de aminorar el problema, además la fruta bajo estas mallas no desarrolla la coloración roja exigida por el mercado y aumenta la labor de poda por el excesivo vigor de las ramas que presentan los árboles cultivados bajo estas condiciones particulares de cultivo (BASTIAS et al., 2015).

El daño por sol se ha clasificado en tres categorías: daño por sol con necrosis, daño por sol con bronceado en la epidermis y un daño a nivel foto-oxidativo. El primero se caracteriza por un daño epidermal causado por la exposición a temperaturas altas por un lapso de alrededor de 10 minutos, lo cual provoca la muerte de las células epidermales del fruto; por otro lado, el segundo daño es ocasionado por la acción conjunta de temperaturas altas y la exposición de frutos a radiación solar, en este caso el bronceado se visualiza en la epidermis al cabo de un par de días después de la exposición a las condiciones mencionadas. Por último, el daño fotoxidativo, el cual es producido por una falta de aclimatación y cuando los frutos son expuestos repentinamente al sol, siendo de distinta intensidad, dependiendo el cultivar (RACSKO y SCHRADER, 2012). Bajo las condiciones climáticas de Chile se ha identificado al menos dos de éstos tipos de daños, el primero causado por altas temperaturas (YURI et al., 2008) y el segundo causado por la combinación de radiación solar y altas temperaturas (BASTIAS et al., 2015).

Es importante, por tanto la búsqueda de tecnologías que permitan aminorar ambos tipos de daño y otorgar mejoras de calidad en los huertos. En la búsqueda de soluciones tecnológicas a los problemas anteriormente señalados, es que en la actualidad existe especial interés por establecer huertos frutales cultivados bajo mallas de color (SHAHAK et al., 2014). Estas mallas están diseñadas con un doble propósito: reducir la incidencia de radiación solar, proteger la fruta de daño por sol y promover respuestas fisiológicas y productivas específicas a través de la modificación en las condiciones de luz (BASTIAS et al., 2015). Así, las mallas en función de su color, deberían afectar en distinta magnitud aspectos ambientales (luz, temperatura y humedad relativa) del huerto, desencadenándose respuestas diferentes en el ámbito productivo, fisiológico y de calidad de frutos en especies frutales (BASTÍAS et al., 2011; LOBOS et al., 2012). Dependiendo del porcentaje de sombreamiento de las mallas, se reduce la cantidad de luz fotosintéticamente activa (PAR), ya sea en el componente de luz directa como difusa que incide sobre el cultivo. Una menor incidencia de radiación PAR bajo las mallas podría tener efectos benéficos al reducir estrés por radiación e hídrico en la planta, además de reducir la variación entre temperatura mínima y máxima dentro del huerto (SHAHAK et al., 2014). Estudios desarrollados en Sudáfrica demostraron que el uso de malla altera condiciones micro-climáticas, tales como temperatura y humedad relativa y, por ende, la actividad estomática y fotosintética de la hoja. Esto último, debido al rol que cumplen estas variables ambientales en la regulación del intercambio gaseoso de $\mathrm{CO}_{2} \mathrm{y} \mathrm{H}_{2} \mathrm{O}$ en la hoja (SMIT et al., 2008). Además, las mallas, dependiendo de color son capaces de modificar la composición del espectro luminoso con un consiguiente efecto sobre aspectos morfológicos regulado por fotorreceptores específicos en la planta, tales como fitocromos (Phy), criptocromos (Cry) y fototropinas (Pho) (SHAHAK et al., 2014; BASTÍAS y CORELLI-GRAPPADELLI, 2012). La acción de éstos fotorreceptores, por ejemplo, está asociado a una reducción del crecimiento de brotes en plantas expuestas a la luz azul $(400-500 \mathrm{~nm})$, regulada por los Cry, como también al alargamiento del entrenudo de brotes en plantas expuestas a una mayor 
proporción de luz roja lejana $(700-800 \mathrm{~nm})$ respecto a la luz roja (600-700 nm), regulada por los Phy (BASTÍAS y CORELLI-GRAPPADELLI, 2012). En otro contexto, aspectos de calidad y condición de la fruta, tales como concentración de azúcar y pigmentos, podrían ser afectados por condiciones de temperatura y luminosidad bajo mallas de color (SHAHAK et al., 2014).

Sin embargo, a la fecha no existe información acerca de cómo estos colores particulares de mallas inciden en el microclima del huerto, el daño por sol y coloración de frutos bajo las condiciones climáticas locales de Chile. Tomando en consideración lo anteriormente mencionado el objetivo de esta investigación fue evaluar el efecto del uso de mallas de color Rojo y Perla sobre el micro-clima, incidencia de quemadura de sol y desarrollo de color de frutos en los cultivares de manzano Fuji y Gala.

\section{MATERIALES Y MÉTODOS}

\section{Diseño experimental y manejos del huerto}

El experimento se llevó a cabo en la Estación Experimental "El Nogal" de la Universidad de Concepción (Campus Chillán), Provincia de Ñuble, Región del Bío Bío (36³6'21" S; 7206'13"' O) durante dos temporadas consecutivas: 2012/2013 y 2013/2014. Durante el periodo de receso invernal del año 2012 se estableció un huerto de manzanos de los cultivares Gala (clon Brookfield) y Fuji (clon Raku Raku), utilizando plantas tipo 'knip' con una cantidad de seis y de cuatro de números de anticipados en 'Gala' y 'Fuji', respectivamente. El huerto se estableció con un marco de plantación de $1,5 \mathrm{~m}$ sobre hilera y 3,5 m entrehilera (1.904 plantas $\mathrm{ha}^{-1}$ ) en una superficie total de $2.315 \mathrm{~m}^{2}$ (496 plantas, distribuidas en cuatro hileras del cultivar Gala y cuatro hileras del cultivar Fuji), incluyendo un 12\% de árboles del cv. Granny Smith como polinizante. Cada hilera de plantación se orientó en dirección norte-sur y los árboles fueron conducidos en sistema 'Slender Spindle', dejando ramas frutales y débiles en la zona alta del árbol (dardos y brindillas) y ramas estructurales y de mayor vigor en la base, de modo tal de generar un diseño en espiral para favorecer la distribución adecuada de la luz solar en la copa y frutos presentes en el árbol. En años sucesivos, y como medida de formación de la estructura mediante poda, se eliminaron aquellas ramas mal ubicadas y con excesivo vigor (crecimiento vertical y poco lignificado).

Una vez finalizada la polinización (cuajado de los frutos), los árboles se cubrieron con mallas monofilamento de color Perla y Roja (ChromatiNet ${ }^{\circledR}$, Polysack Industries, Negev, Israel) que aportan un $20 \%$ de sombreamiento, dejando árboles sin malla (ambiente) como control. Las mallas fueron instaladas a una altura de 3,5 metros sobre una estructura confeccionada con polines de pino impregnado de 4,5 metros de altura (insertos en el suelo a una profundidad de un metro) y alambres acerados ACC (alto contenido de carbono) de $8 \mathrm{~mm}$ $(17 / 15)$ de diámetro (Figura 1). Las características de trasmisión espectral de la luz de las mallas empleadas fueron previamente establecidas con un espectroradiómetro LI-1800 (LI-COR, Lincoln, NE, USA). La malla Roja incrementa la transmisión de luz en el rango de longitud de onda de $600-1100$ $\mathrm{nm}$, es decir en los espectros de luz roja (600 - 700 $\mathrm{nm})$, rojo lejano (700 -800 nm) e infra-rojo cercano $(800-1100 \mathrm{~nm})$, mientras que la malla Perla no altera el espectro de transmisión de luz en el rango evaluado (Figura 2).

En total se cubrió 144 árboles de ambos cultivares por tratamiento y el experimento fue conducido en un diseño completamente al azar de cinco repeticiones, de dos árboles cada una (10 árboles/tratamiento). En cada repetición se evaluaron los árboles de las dos hileras centrales de cada parcela, la cual constaba de cuatro hileras por cultivar, para evitar el efecto del sombreamiento y de difusión de la luz entre mallas o entre hileras.

El suministro de agua al huerto se realizó mediante riego por goteo, con un lateral de polietileno (PE) de $16 \mathrm{~mm}$ por hilera, y dos emisores por planta, Netafim autocompensados, a una distancia de 0,5 $\mathrm{m}$, con caudal de $2 \mathrm{~L} \mathrm{~h}^{-1}$ y presión de 10 metros de columna de agua (m.c.a.). La recomendación de riego se determinó en función del estado fenológico, y la tasa de aplicación de agua de los goteros (QUEZADA et al., 2011). El riego se aplicó entre los meses de noviembre a marzo de 2012/13 y 2013/14, con una frecuencia de riego entre 1 a 3 días y tiempos de riego de 2 a $4 \mathrm{~h}$ con un volumen aplicado de 5.582 $\mathrm{m}^{3} \mathrm{ha}^{-1}$ en las temporadas 2012-13 y 2013-2014, respectivamente.

\section{Condiciones ambientales del huerto}

Bajo las condiciones de malla Roja, Perla y el control (ambiente) se llevó a cabo un registro continuo de temperatura $\left({ }^{\circ} \mathrm{C}\right)$ y humedad relativa del aire (\%) a intervalos de 15 minutos con dispositivos Hobo data logger (HOBO Pro HR/ Temp, Onset Computer Corporation) instalados en cada tratamiento. Las variaciones de la intensidad de radiación fotosintéticamente activa total $\left(\mathrm{PAR}_{\text {total }}\right)$ y difusa $\left(\mathrm{PAR}_{\text {difusa }}\right)$, expresada en flujo de fotones 


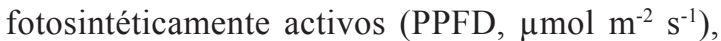
se determinaron con el sensor quantum LI-191SA conectado a un datalogger LI-1400 (LICOR, Lincoln, NE, USA). Para obtener los valores de PAR $_{\text {difusa }}$ el sensor quantum fue localizado en una estructura que simula la trayectoria del sol (Figura 3), generando una sombra proyectada por una esfera de goma negra $(32,57 \mathrm{~cm}$ de diámetro), la que se desplaza sobre la base según el horario de la medición (9:00; 11:00; 13:00; 15:00 y 17:00 horas).

Paralelamente a las mediciones de PAR, se registró la temperatura superficial de los frutos utilizando un termómetro infra-rojo (Extech Instruments Corporation, U.S.A.). Este registro se realizó en un total de seis frutos por tratamiento ubicados en la posición sur-poniente de los árboles y una distancia de $20 \mathrm{~cm}$ entre el fruto y el sensor.

\section{Daño por sol y coloración de frutos}

El daño por sol en frutos se cuantificó visualmente, y de acuerdo a la siguiente escala (YURI et al., 2010): Fruta sana (sin daño aparente), daño leve (zona epidérmica con coloración amarillo tenue), daño moderado (zona epidérmica con coloración amarillo-rojiza intensa), daño severo (zona epidérmica con coloración parda y daño lenticelar), expresando los daños en porcentaje (\%) de fruta dañada. Paralelamente, la totalidad de frutos cosechados por planta fueron analizados con un equipo de colorimetría Color Hunter Lab, el cual determinó los índices de color en la epidermis en seis puntos diferentes del fruto, situados en la zona ecuatorial. Cada punto fue registrado según la escala internacional de CIELAB (Commision Internationale de L'Eclairage, CIE, 2004) a intervalos de aproximadamente 60 segundos, tiempo requerido para girar el fruto. El espectro de luz que atraviesa la superficie epidermal del fruto representa, en coordenadas cartesianas, valores de $\mathrm{L}^{*}, \mathrm{a}^{*}, \mathrm{~b}^{*}$ y un ángulo HUE $\left(\mathrm{h}^{\circ}\right)$ (ecuación $\mathrm{n}^{\circ} 1$ ) que determina la longitud de onda en la que se realiza la medición (CIE, 2004). Así se tiene una inclinación de 0-45 para cada parámetro, en planos positivos y negativos, en donde L* representa luminosidad y sombra, a* (rojo) y $-\mathrm{a}^{*}$ (verde), $\mathrm{b}^{*}$ (amarillo) y $-\mathrm{b}^{*}$ (azul).

Ecuación 1: $\mathrm{h}^{\circ}=\tan ^{-1}\left(\mathrm{~b}^{*} / \mathrm{a}^{*}\right)($ LANCASTER et al., 1997)

Donde:

$\mathrm{h}^{\circ}$ : Ángulo HUE, $\tan ^{-1}$ : Tangente.

\section{Análisis estadístico}

Los datos obtenidos fueron sometidos a un análisis de varianza ANOVA $(p<0,05, p<0,01)$ para luego determinar la diferencia de medias mediante el test Student Newman Keuls. Además, los valores expresados en porcentajes fueron normalizados mediante la fórmula del arcosen $\sqrt{ }(x / 100)$, en donde $x$ corresponde al valor porcentual. Todos estos análisis se realizaron con el programa estadístico Statgraphics Centurion XVI (StatPoint Technologies, 2009).

\section{RESULTADOS Y DISCUSION}

\section{Condiciones micro-climáticas}

La Figura 4 muestra la variación diaria en la incidencia de $\mathrm{PAR}_{\text {total }}$ y $\mathrm{PAR}_{\text {difusa }}$ para las temporadas 2012/13 y 2013/14. En ambas temporadas productivas la intensidad de luz, expresada en PAR $_{\text {total }}$ fue mayor $(p<0,01)$ en el tratamiento control, respecto a los tratamientos de malla Roja y Perla (Fig. 4 A y B). No obstante, la capacidad de transmisión de luz varió entre temporadas y tipo de malla. Para la temporada 2012/13, las mallas Roja y Perla redujeron en promedio un 25 y $20 \%$ la cantidad diaria de $\mathrm{PAR}_{\text {total }}$, respectivamente (Figura 4A), mientras que durante la siguiente temporada estas mallas redujeron la cantidad de PAR $_{\text {total }}$ transmitida en un 17 y $13 \%$, respectivamente (Figura 4B). Las razones de estas diferencias pueden estar asociadas a modificaciones en las propiedades radiométricas de la malla. Se ha mencionado en previos estudios que la efectividad de transmisión de radiación PAR de las mallas de color puede variar en un rango de $6-10 \%$ por factores externos, tales como la pérdida de la estabilidad de los pigmentos de la malla frente a los altos niveles de radiación UV, acumulación de polvo que modifican el factor de sombreamiento y el incremento de la tensión de las tramas y urdimbre de los hilos por efecto del trabajo de apertura y cierre de mallas o variaciones en la temperatura ambiental (SHAHAK et al., 2004).

Por otra parte, la cantidad de PAR transmitida bajo mallas varía ampliamente dependiendo del diseño y color. Solomakhin y Blanke (2008), demostraron una variación de aproximadamente un $5 \%$ en la transmisión de la radiación PAR, dependiendo de la distancia y color de hilos utilizado en el diseño de la malla. Con el uso de malla sombra Roja y Azul la $\mathrm{PAR}_{\text {total }}$ disminuyó en un $27 \%$, mientras que con la malla Gris fue aún mayor, en un 37\% (BASTÍAS et al., 2011). Lo anterior coincide con los resultados de nuestro estudio, en donde la cantidad de luz PAR disponible bajo la malla Perla fue en promedio, para ambas temporadas, un $7 \%$ 
superior a la registrada bajo la malla Roja (Fig. 4 A y B).

Una mayor PAR $_{\text {total }}$ bajo la malla Perla podría indicar una mayor actividad fotosintética en la planta, asociada a un aumento del contenido de clorofila en las hojas (DO AMARANTE et al., 2011), puesto que la radiación y su intensidad están en relación directa con la actividad de los fotosistemas y su inhibición en la piel de la fruta por un exceso de radiación u otro factor ambiental como altas temperaturas (CHEN et al., 2008). La menor PAR bajo malla Roja respecto a la malla Perla (Fig. 4 A y B) podría explicarse por las longitudes de onda que transmite esta malla (Fig. 2), en que se demuestra una reducción de la transmisión de la luz de las longitudes de onda menores a $580 \mathrm{~nm}$, es decir, afectando la radiación de la luz azul, que es componente importante del espectro de luz PAR (BASTÍAS y CORELLI-GRAPPADELLI, 2012). Otra explicación a este fenómeno es el efecto del color de la malla sobre la proporción de luz difusa. En ambas temporadas bajo el tratamiento cubierto con malla Perla se registró una mayor cantidad de luz difusa $(\mathrm{p}<0.01)$, siendo superior en un $21 \%$ para la temporada $2012 / 13$ y un $7 \%$ para la temporada 2013/14, con respecto a la malla Roja (Fig. 4 C y D). Según Oren Shamir et al (2001), dependiendo del color de las mallas, puede aumentar la difusión de la luz en hasta un $50 \%$, siendo aquellas mallas fabricadas con hilos de colores más claro las que aportan una mayor proporción de luz difusa a las plantas.

Recientes estudios desarrollados en arándanos cubiertos con túneles de filme de polietileno, demostraron que bajo esta condición de cultivo la mayor proporción de luz difusa fue estrechamente relacionada con un aumento en la conductancia estomática de la hoja (RETAMAL-SALGADO et al., 2015). El efecto positivo de la radiación difusa sobre el intercambio gaseoso de la planta puede ser atribuido a que estas condiciones micro-climáticas de luz resultan favorables en la reducción de la temperatura de la hoja promoviendo así la apertura estomática (URBAN et al., 2007).

La Figura 5 muestra las diferencias de temperatura y humedad relativa del aire registradas bajo los distintos tipos de mallas evaluadas. Aunque las diferencias registradas fueron mínimas, se observó una reducción de la temperatura del orden de $0,5^{\circ} \mathrm{C}$ con la malla Perla en comparación a la malla Roja y un aumento de la humedad relativa del orden del 3-5\%. Estos resultados están en concordancia con aquellos obtenidos en Israel y España, quienes determinaron una disminución de $1-5^{\circ} \mathrm{C}$ bajo malla, respectivamente, y un aumento de la humedad relativa del orden del 3-10\% (SHAHAK et al, 2004; IGLESIAS y ALEGRE, 2006).

Por otra parte, para ambas temporadas la temperatura superficial de la epidermis de manzanas también se vio alterada por efecto del color de la malla al menos en el cv. Gala (Figura 6 A y B). En frutos expuestos al sol, la temperatura fue en promedio $2,5^{\circ} \mathrm{C}$ y $1,5^{\circ} \mathrm{C}$ inferior bajo malla Perla en comparación al control, mientras que la malla Roja no mostró un efecto significativo sobre la temperatura del fruto, de hecho esta resultó ser levemente mayor a la situación control, sin malla (Figura 6 A y B). Esta disminución en la temperatura se debería al efecto de la mayor proporción de luz difusa que aporta la malla Perla (Figura 4 C y D), cuyo componente de luz promueve una reducción en la temperatura de los tejidos vegetales (URBAN et al., 2007).

En el cultivar Fuji (Fig. $6 \mathrm{C}$ y D) la temperatura de los frutos entre tratamientos no fue diferente $(p>0.05)$ para ambas temporadas. Este comportamiento diferente podría deberse a que las mediciones de temperatura se tomaron en cultivares con distinta fecha de maduración; 'Gala' es una variedad de cosecha temprana y 'Fuji' de cosecha tardía. Se ha reportado que en la medida que la manzana crece y madura en el árbol su capacidad de transpiración disminuye notablemente, por lo que también disminuye su capacidad de regular la temperatura de la epidermis bajo condiciones de extrema radiación solar (MORANDI et al., 2012), por lo que el efecto de las mallas sobre la temperatura de frutos sería más marcado en este caso en 'Gala', debido a que las mediciones se tomaron para este cultivar en frutos con estado de madurez más avanzada y por tanto menor capacidad de transpiración (Fig. 6 A y B). Esto ha sido avalado por estudios realizados en Chile en que se demostró que los daños causados en la epidermis por exposición de frutos a altas temperaturas durante la temporada productiva (3-4 meses) dependen de si el cultivar es temprano o tardío (YURI et al., 2008). Otros autores consideran que la combinación de la temperatura de los frutos y la radiación incidente sobre éstos son responsables de una mayor o menor incidencia de daño por sol en la epidermis de los frutos (VALENZUELA y MUÑOZ, 2011; RACSKO y SCHRADER, 2012). En Israel se ha demostrado que, dependiendo del color de la malla, podría reducirse la temperatura del aire de $1-5^{\circ} \mathrm{C}$, no obstante no existen datos sobre el efecto de mallas de color en la temperatura de los frutos (SHAHAK et al., 2004). 


\section{Golpe de sol y desarrollo de color}

Durante la temporada 2012/13, tanto la malla Perla como Roja redujeron significativamente la cantidad de fruta dañada por golpe de sol, y para ambos cultivares evaluados (Tablas 1 y 2). En promedio ambas mallas redujeron la incidencia de daño por sol en $26 \%$ para 'Gala' (Tabla 1), mientras que en 'Fuji' la reducción de fruta dañada por sol bajo ambas mallas fue de un 44\% (Tabla 2). Esto corrobora el efecto positivo de la reducción en la intensidad de radiación (Figura 4) sobre una menor incidencia de daño por quemadura de sol (VALENZUELA y MUÑOZ, 2011). No obstante, durante la siguiente temporada (2013/14) no se observó un efecto significativo de las mallas sobre la reducción de daño por sol en la fruta (Tablas 2 y 3). De hecho en el cultivar Fuji el uso de malla Roja incrementó la cantidad de fruta con daño por sol de tipo moderado (Tabla 2). Tal como fuera discutido anteriormente, esta respuesta se debería a la pérdida en la efectividad de las mallas en la capacidad de reducir la transmisión de radiación PAR (Figura 4 A y B). Otra razón puede deberse a variaciones en las condiciones climáticas que existieron entre ambas temporadas. Bajo las condiciones de Chile se ha determinado que el daño por sol en manzanas se manifiesta cuando la temperatura del aire supera $\operatorname{los} 29^{\circ} \mathrm{C}$ (YURI et al., 2008). Según los registros de la estación meteorológica ubicada en el lugar del ensayo, entre los meses de diciembre a marzo de la temporada 2012/13 existieron 42 días en los cuales las temperaturas máximas superaron este umbral de $29^{\circ} \mathrm{C}$, mientras que durante la temporada 2013/14 la cantidad eventos con temperaturas sobre este este umbral fue muy superior y alcanzó a los 54 días (Tabla 3), es decir condiciones más extremas de calor que estarían explicando la menor efectividad de las mallas en el control de daño por sol para dicha temporada (Tablas 1 y 2). Cabe destacar que los niveles de radiación solar para el periodo no variaron en forma sustancial entre ambas temporadas y alcanzaron un rango promedio de $24-25 \mathrm{MJ} \mathrm{m}^{-2}$ día $^{-1}$ (Tabla 3), confirmando lo determinante que puede resultar el incremento de temperatura como causa principal de la generación de daño por sol en manzanas bajo las condiciones climáticas locales de Chile (YURI et al., 2008).

Los datos obtenidos del análisis colorimétrico de los frutos muestran que para ambos cultivares el valor del parámetro $a^{*}$ fue significativamente $(p<0,05)$ superior bajo malla Perla y control (sin malla) en comparación a la malla Roja, durante la primera temporada para el cultivar Gala (Tabla 4) y para ambas temporadas en el cultivar Fuji (Tabla 5).
Un incremento en el parámetro a* está estrechamente relacionado con el aumento de coloración roja y síntesis de antocianinas en la piel de los frutos (LANCASTER et al., 1997). El efecto positivo de la malla Perla sobre la mayor coloración de frutos, puede ser explicado por la mayor proporción de luz difusa que se generó bajo esta malla (Figura $4 \mathrm{C} \mathrm{y}$ D), lo que favorece la mayor disponibilidad de luz en sectores más sombríos del árbol (SHAHAK et al., 2014), favoreciendo la síntesis de antocianinas en frutos, ya sea directamente como mecanismo de foto-protección (FELICETTI y SCHRADER, 2008; CHEN et al., 2008), o indirectamente al favorecer una mayor disponibilidad de carbohidratos producto del incremento en la capacidad fotosintética en las hojas de los árboles expuestas a una mayor proporción de luz difusa (BASTIAS et al., 2012). 
TABLA 1- Efecto del uso de mallas de color Perla y Roja sobre la incidencia de daño por sol en manzanas 'Gala'. Chillá

\begin{tabular}{ccccc}
\hline \multirow{2}{*}{ Tratamientos } & \multicolumn{4}{c}{ Categoría de daño por sol $(\%)$} \\
\cline { 2 - 5 } & Sana & Leve & Moderado & Severo \\
\hline 2012/13 & & & & \\
Perla & $92,7 \mathrm{a}$ & $7,3 \mathrm{ab}$ & $0,0 \mathrm{a}$ & $0,0 \mathrm{a}$ \\
Roja & $96,6 \mathrm{a}$ & $3,4 \mathrm{a}$ & $0,0 \mathrm{a}$ & $0,0 \mathrm{a}$ \\
Control & $68,9 \mathrm{~b}$ & $14,9 \mathrm{~b}$ & $12,1 \mathrm{~b}$ & $4,1 \mathrm{~b}$ \\
Significancia & $* *$ & $*$ & $* *$ & \\
& & & & \\
$2013 / 14$ & & & & $0,0 \mathrm{a}$ \\
Perla & 81,6 & 18,4 & 0,0 & $0,0 \mathrm{a}$ \\
Roja & 67,0 & 27,4 & 5,5 & $5,0 \mathrm{~b}$ \\
Control & 64,2 & 18,7 & 12,1 & $*$ \\
Significancia & N.S. & N.S. & N.S. & \\
\hline
\end{tabular}

*; **: significancia a $\mathrm{p}<0.05$ y 0.01 , respectivamente.

TABLA 2 - Efecto del uso de mallas de color Perla y Roja sobre la incidencia de daño por sol en manzanas 'Fuji'.

\begin{tabular}{lcccc}
\hline \multirow{2}{*}{ Tratamientos } & \multicolumn{4}{c}{ Categoria de golpe de sol (\%) } \\
\cline { 2 - 5 } & Sana & Leve & Moderado & Severo \\
\hline $\mathbf{2 0 1 2 / 1 3}$ & & & & \\
Perla & $70,1 \mathrm{a}$ & 24,8 & $5,0 \mathrm{a}$ & 0,0 \\
Roja & $58,3 \mathrm{a}$ & 36,6 & $5,0 \mathrm{a}$ & 0,0 \\
Control & $19,8 \mathrm{~b}$ & 18,0 & $24,0 \mathrm{~b}$ & 38,0 \\
Significancia & $* *$ & N.S. & $* *$ & $* *$ \\
& & & & \\
$\mathbf{2 0 1 3 / 1 4}$ & & & & \\
Perla & 38,6 & 40,2 & $14,4 \mathrm{ab}$ & $8,3 \mathrm{a}$ \\
Roja & 34,8 & 34,0 & $27,6 \mathrm{~b}$ & $3,3 \mathrm{a}$ \\
Control & 39,0 & 27,9 & $6,8 \mathrm{a}$ & $25,9 \mathrm{~b}$ \\
Significancia & N.S. & N.S. & $*$ & $*$ \\
\hline
\end{tabular}

*; **: significancia a $\mathrm{p}<0.05$ y 0.01 , respectivamente.

TABLA 3 - Registro de condiciones de temperatura y radiación solar entre los meses de diciembre y marzo para las temporadas en que se efectuaron los ensayos. Chillán, Chile.

\begin{tabular}{lcc}
\hline Temporada & Temperatura máxima $>29^{\circ} \mathrm{C}$ (días) & $\begin{array}{c}\text { Radiación solar media } \\
\left(\mathrm{MJ} \mathrm{m}^{-2} \text { día }^{-1}\right)\end{array}$ \\
\hline $2012 / 13$ & 42 & 25,3 \\
$2013 / 14$ & 54 & 24,3 \\
\hline
\end{tabular}


TABLA 4- Parámetros de color en frutos de manzana 'Gala' cubiertos por mallas Perla y Roja.

\begin{tabular}{lcccc}
\hline \multirow{2}{*}{ Tratamientos } & \multicolumn{4}{c}{ Parámetros de color } \\
\cline { 2 - 5 } & $\mathrm{L}^{*}$ & $\mathrm{a}^{*}$ & $\mathrm{~b}^{*}$ & Hue \\
\hline $\mathbf{2 0 1 2 / 1 3}$ & & & & \\
Perla & 48,1 & $29,3 \mathrm{ab}$ & 19,6 & 31,3 \\
Roja & 47,3 & $28,0 \mathrm{~b}$ & 19,2 & 32,2 \\
Control & 46,4 & $31,5 \mathrm{a}$ & 18,9 & 28,6 \\
Significancia & N.S. & $*$ & N.S. & N.S. \\
& & & & \\
$\mathbf{2 0 1 3 / 1 4}$ & & & & \\
Perla & 41,2 & 34,4 & 18,3 & 25,7 \\
Roja & 40,7 & 35,9 & 18,4 & 24,9 \\
Control & 38,9 & 35,2 & 17,9 & 24,5 \\
Significancia & N.S. & N.S. & N.S. & N.S. \\
\hline
\end{tabular}

*; **: significancia a $\mathrm{p}<0.05$ y 0.01 , respectivamente.

TABLA 5- Parámetros de color en frutos de manzana 'Fuji' cubiertos por mallas Perla y Roja.

\begin{tabular}{lcccc}
\hline \multirow{2}{*}{ Tratamientos } & \multicolumn{4}{c}{ Parámetros de color } \\
\cline { 2 - 5 } & $\mathrm{L}^{*}$ & $\mathrm{a}^{*}$ & $\mathrm{~b}^{*}$ & Hue \\
\hline $\mathbf{2 0 1 2 / 1 3}$ & & & & \\
Perla & 47,1 & $24,4 \mathrm{a}$ & $21,7 \mathrm{a}$ & 38,6 \\
Roja & 48,4 & $21,3 \mathrm{~b}$ & $21,7 \mathrm{a}$ & 42,5 \\
Control & 47,7 & $23,8 \mathrm{a}$ & $23,9 \mathrm{~b}$ & 41,4 \\
Significancia & N.S. & $*$ & $* *$ & N.S. \\
& & & & \\
$\mathbf{2 0 1 3 / 1 4}$ & & & & $43,2 \mathrm{a}$ \\
Perla & $47,4 \mathrm{a}$ & $24,2 \mathrm{a}$ & $23,0 \mathrm{~b}$ & $53,2 \mathrm{~b}$ \\
Roja & $50,7 \mathrm{~b}$ & $18,6 \mathrm{~b}$ & $25,6 \mathrm{a}$ & $43,1 \mathrm{a}$ \\
Control & $46,6 \mathrm{a}$ & $23,6 \mathrm{a}$ & $22,5 \mathrm{~b}$ & $* *$ \\
Significancia & $* *$ & $* *$ & $* *$ & \\
\hline
\end{tabular}

*; **: significancia a $\mathrm{p}<0.05$ y 0.01 , respectivamente. 


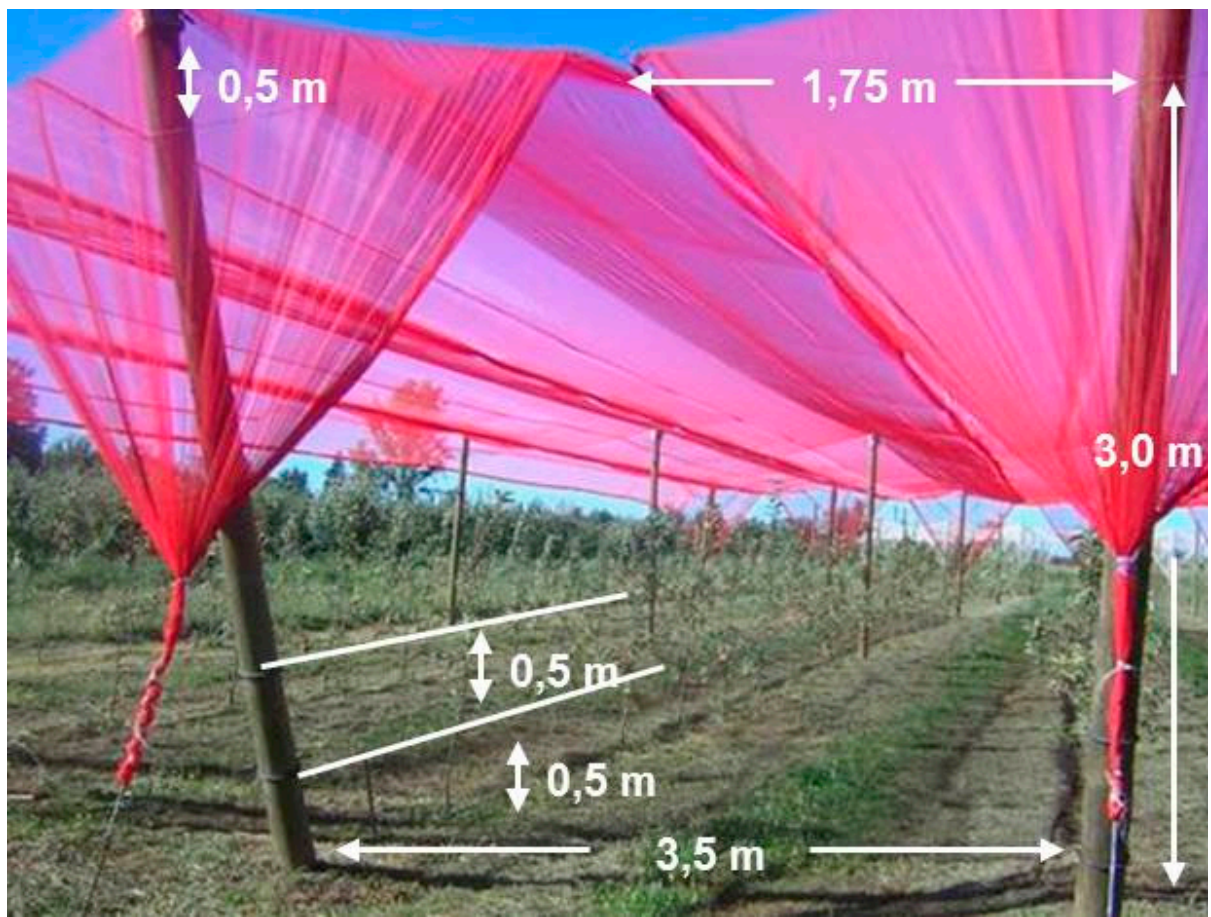

FIGURA 1- Estructura utilizada para la instalación de mallas de color Roja y Perla en huertos de manzanos, Chillán, Chile.

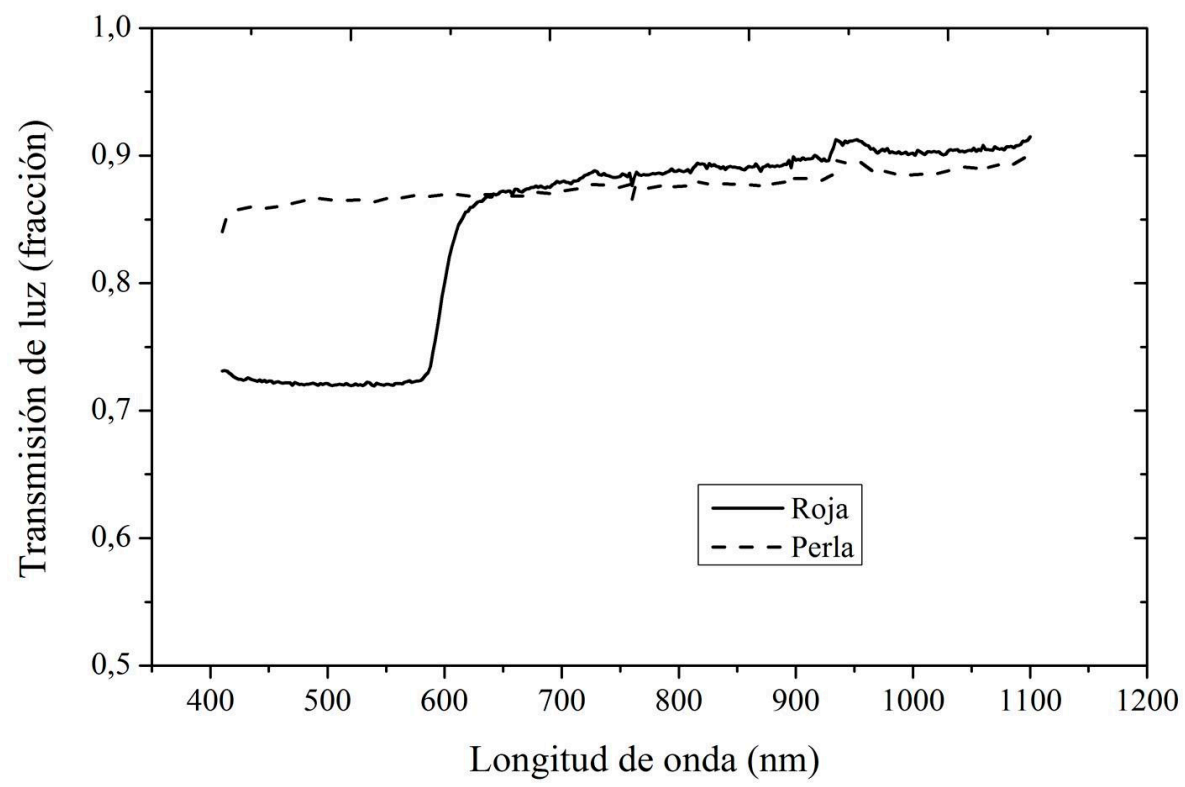

FIGURA 2- Patrón de transmisión espectral de luz de mallas Perla y Roja utilizadas para cubrir manzanos. 


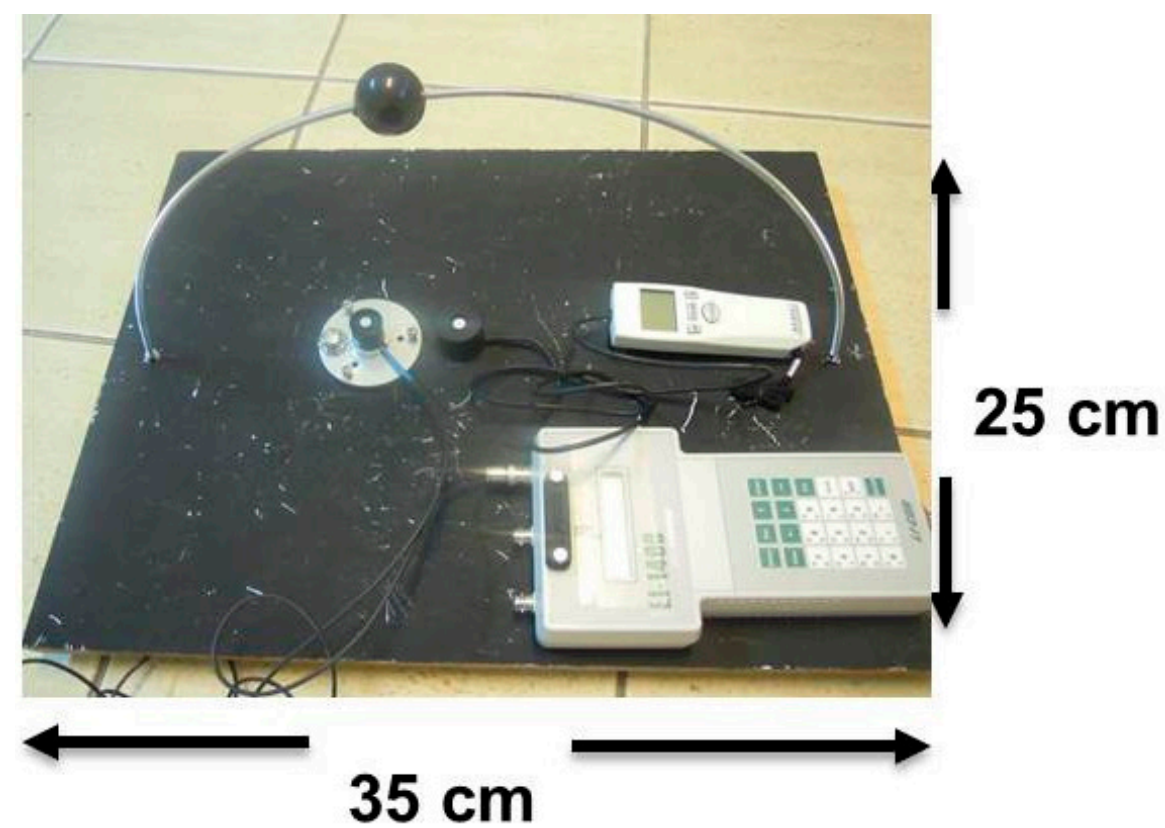

FIGURA 3- Método empleado para determinar valores de PAR difusa.

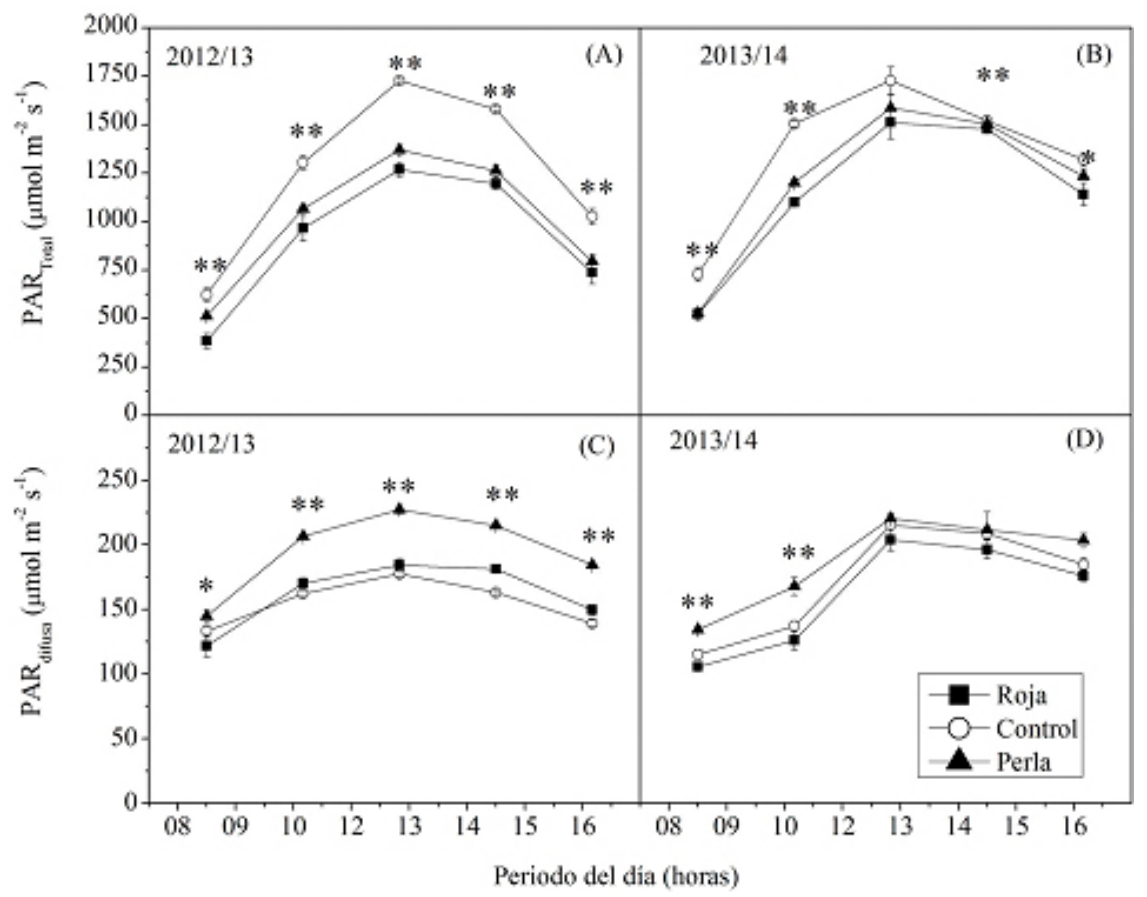

FIGURA 4-Variación diaria en la incidencia de radiación fotosintéticamente activa (PAR) total (A y B) y difusa (C y D) para huertos de manzano cubiertos por mallas de color Perla y Roja. Chillán, Chile. Temporadas 2012/13 y 2013/14.

*; **: significancia a $\mathrm{p}<0,05$ y 0,01 , respectivamente. Cada punto representa las medias $\pm \mathrm{ES}$ de seis muestreos. 


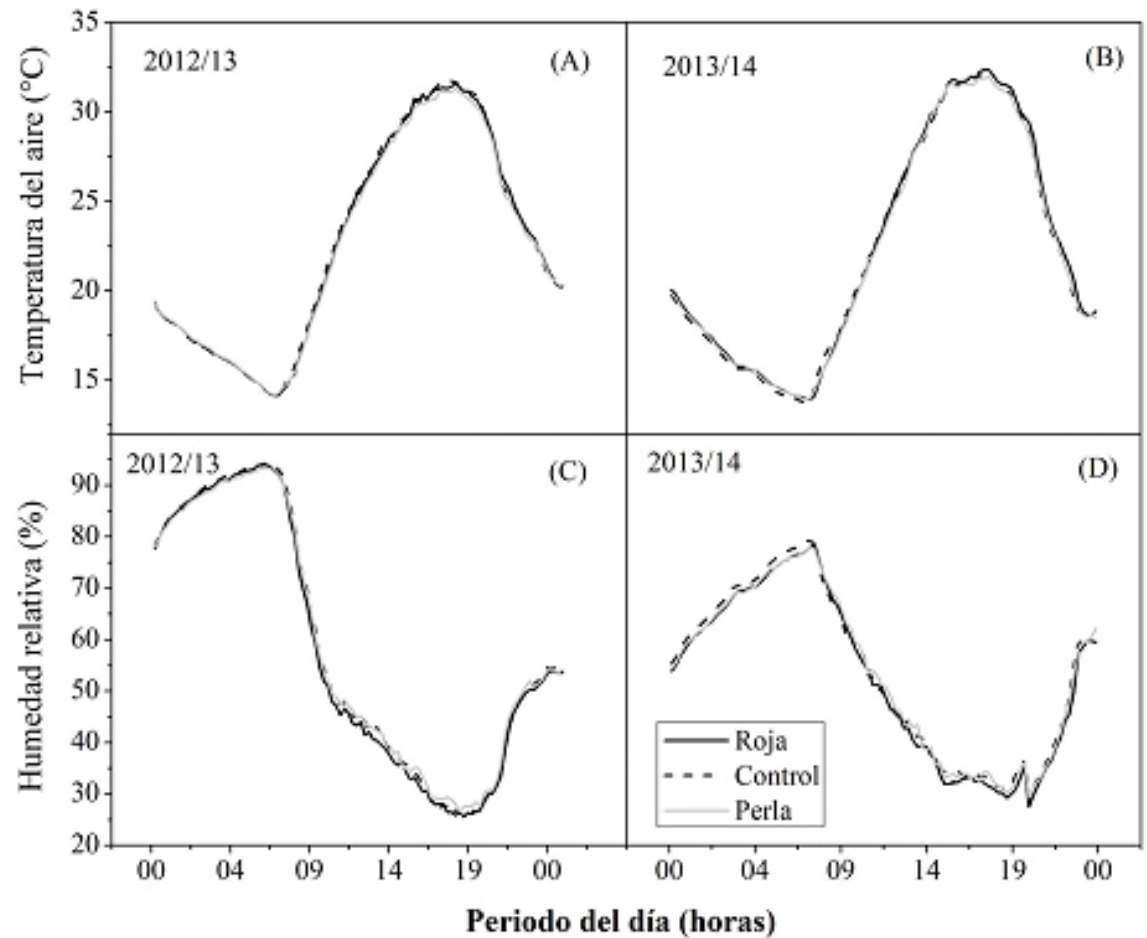

FIGURA 5- Patrón diurno de temperatura del aire (A y B) y humedad relativa (C y D) en huertos de manzanos cubiertos por malla Perla y Roja. Chillán, Chile. Temporadas 2012/13 y 2013/14.

*; **: significancia de $\mathrm{p}<0.05$ y 0.01 , respectivamente.

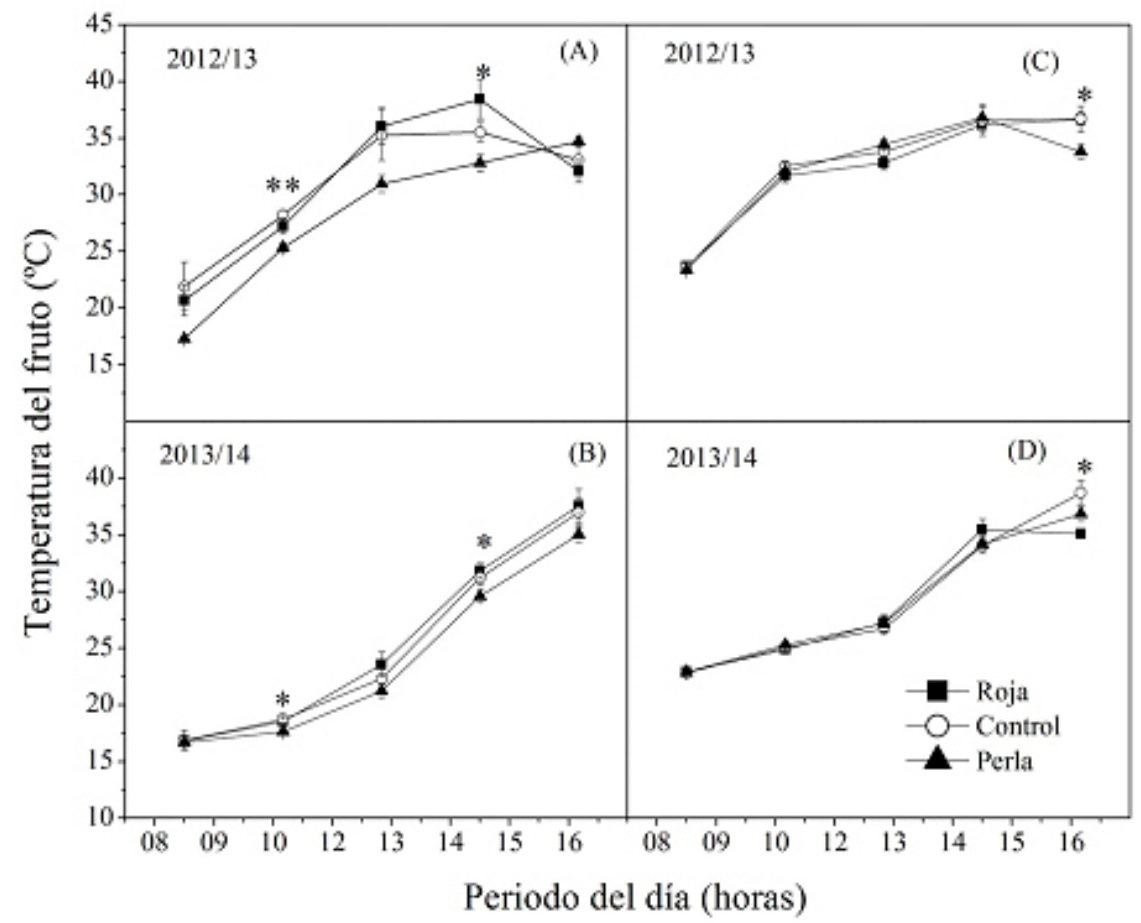

FIGURA 6- Patrón diurno de temperatura de frutos en huertos de manzanos 'Gala' (A y B) y 'Fuji' (C y D) cubiertos por mallas Perla y Roja. Chillán, Chile. Temporadas 2012/13 y 2013/14. 


\section{CONCLUSIONES}

Las mallas Perla y Roja alteran diferencialmente las condiciones lumínicas en huertos de manzanos.

La malla Perla incrementa la disponibilidad de luz, especialmente la proporción de luz difusa, con efectos positivos en la disminución de la temperatura y mejora de coloración de frutos.

Bajo las condiciones climáticas de Chile, la efectividad de las mallas Perla y Roja en el control de daño por sol varía ampliamente dependiendo de las condiciones de temperatura y de la capacidad del material de la malla para filtrar la radiación.

\section{AGRADECIMIENTOS}

El presente estudio fue realizado, gracias a los aportes del proyecto "Mejorando el uso de la luz a través de mallas foto-selectivas en huertos de manzano: Estudio de mecanismos fisiológicos y efectos sobre la producción y calidad de frutos", financiado por la Dirección de Investigación de Universidad de Concepción, Chile.

\section{REFERENCIAS}

BAEZA, C.; NAVARRO, M. Manzana chilena ¿Dónde estamos parados? Revista Frutícola, Curicó, v.2, p.4-5, 2008.

BASTÍAS, R.M.; CORELLI-GRAPPADELLI, L. Light quality management in fruit orchards: physiological and technological aspects. Chilean Journal of Agricultural Research, Chillán, v.72, n.4, p.574-581, 2012.

BASTÍAS, R.M.; LEYTON, M.J.; UMANZOR, C.; VALENZUELA, R.; CORELLI-GRAPPADELLI, L. Uso de mallas en huertos de manzanos: Parte I. Consideraciones en el diseño, propiedades radiométricas y respuestas de interés agronómico. Revista Frutícola, Curicó, v.37, p.32-37, 2015.

BASTÍAS, R.M.; LOSCIALE, P.; CHIECO C.; ROSSI F.; CORELLI-GRAPPADELLI, L. Physiological aspects affected by photoselective nets in apples: preliminary studies. Acta Horticulturae, The Hague, v.907, p.217-220, 2011.
CHEN, L.; LI, P.; CHENG, L. Effects of high temperature coupled with high light on the balance between photooxidation and photoprotection in the sun-exposed peel of apple. Planta, Berlin, v.228, p.745-756, 2008.

COMMISSION INTERNATIONALE DE L'ECLAIRAGE, Colourimetry. Publication CIE, Vienna,152, 1986.

DO AMARANTE, C.V.T.; STEFFENS, C.A.; ARGENTA, L.C. Yield and fruit quality of 'Gala' and 'Fuji' apple trees protected by white anti-hail net. Scientia Horticulturae, Amsterdam, v.129, n.1, p.79-85, 2011.

FELICETTI, D.A.; SCHRADER, L.E. Changes in pigment concentrations associated with the degree of sunburn browning of 'Fuji' apple. Journal of the American Society for Horticultural Science, Washington, v.133, n.1, p.27-34, 2008.

IGLESIAS, I.; ALEGRE, S. The effect of anti-hail nets on fruit protection, radiation, temperature, quality and profitability of 'Mondial Gala' apples. Journal of Applied Horticulture, Lucknow, v.8, n.2, p.91-100, 2006.

LANCASTER, J. E.; LISTER, C. E.; REAY, P. F.; TRIGGS, C. M. Influence of pigment composition on skin color in a wide range of fruit and vegetables. Journal of the American Society for Horticultural Science, Alexandria, v.122, n. 4, p.594-598, 1997.

LOBOS, G.A.; RETAMALES, J.B.; HANCOCK, J.F.; FLORE, J.A.; COBO, N.; DEL POZO, A. Spectral irradiance gas exchange characteristics and leaf traits of Vaccinium corymbosum L. 'Elliott' grown under photo-selective nets. Environmental and Experimental Botany, Netherlands, v.75, p.142-149, 2012.

MORANDI, B.M.; ZIBORDI, P.; LOSCIALE, L.; MANFRINI, R.M.; BASTIAS, L.; CORELLI GRAPPADELLI. Apple and peach: a different role for fruit transpiration?. Acta Horticulturae, The Hague, v.932, p.213-218, 2012. 
OREN-SHARIM, M.; GUSSAKOVSKY, E.; SHPIEGEL, E.; NISSIN-LEVI, A.; RATNER, K.; OVADIA, R.; GILLER, Y.; SHAHAK, Y. Colored shade nets can improve the yield and quality of green decorative branches of Pittosporum variegatum. Journal of Horticultural Science and Biotechnology, Ashford, v.76, p.353-361, 2001.

QUEZADA, C.; SOLIS, E.; VENEGAS, A.; FAÚNDEZ, M. Efecto de 4 niveles de aplicación de agua en rendimiento y calidad de un huerto de manzanos (Malus domestica Bork) Fuji bajo riego por goteo. Agro Ciencia, Revista Chilena Ciencias Agropecuarias, Chillán, v.27, p.65-75, 2011.

RACSKO, J.; SCHRADER, L.E. Sunburn of apple fruit: Historical background, recent advances and future perspectives. Critical Reviews in Plant Sciences, United Kingdom, v.31, n.6, p.455-504, 2012.

RETAMAL-SALGADO, J.; BASTÍAS, R.M.; WILCKENS, R.; PAULINO, L. Influence of microclimatic conditions under high tunnels on the physiological and productive responses in blueberry cv. O'Neal. Chilean Journal of Agricultural Research, Chillán, v.75, n.3, p.291-297, 2015.

SHAHAK, Y. Photo-selective netting: An overview of the concept, research and development and practical implementation in agriculture. Acta Horticulturae, The Hague, v.1015, p.155- 162, 2014.

SHAHAK, Y.; GUSSAKOVSKY, E.E.; GAL, E.; GANELEVIN, R. Colour nets: Crop protection and light quality manipulation in one technology. Acta Horticulturae, The Hague, v.659, p.143-151, 2004.

SMIT, A.; STEYN, W.J.; WAND, S.J.E. Effects of Shade Netting on Gas Exchange of Blushed Apple Cultivars. Acta Horticulturae, The Hague, v.772, p.73-80, 2008.
SOLOMAKHIN, A.; BLANKE, M.M. Coloured hailnets alter light transmission, spectra and phytochrome, as well as vegetative growth, leaf chlorophyll and photosynthesis and reduce flower induction of apple. Plant Growth Regulation, Dordrecht, v.56, p.211-218, 2008.

STATPOINT TECHNOLOGIES. Statgraphics. Warrenton: StatPoint Technologies, 2009.

URBAN, O.; JANOUS, D.; ACOSTA, M.; CZERNY, R.; MARKOVA, I.; NAVRATIL, M. Ecophysiological controls over the net ecosystem exchange of mountain spruce stand. Comparison of the response in direct vs. diffuse solar radiation. Global Change Biology, Oxford, v.13, p.157-168, 2007.

VALENZUELA, L.; MUÑOZ, C. Daño por sol y color rojo en manzano: Diagnostico y orientación óptima de los huertos. Revista Frutícola, Curicó, v.2, p.10-19, 2011.

YURI, J.A.; LEPE, V.; SEPULVEDA, A.; BASTIAS, R.; MOGGIA, C.; GONZALEZ, J.: LOBOS, G.; GLENN, M. Sunburn on apples: Fifteen years of research in Chile supports heat as the main cause of damage. HortScience, Alexandria, v.43, n.4, p.1110-1111, 2008.

YURI, J.A.; NEIRA, A.; QUILODRAN, A.; RAZMILIC, I.; MOTOMURA, Y.; TORRES, C.; PALOMO, I. Sunburn on apples is associated with increases in phenolic compounds and antioxidant activity as a function of the cultivar and areas of the fruit. Journal of Food, Agriculture \& Environment, Helsinki, v.8, n.2, p.920-925, 2010. 